\title{
Article
}

\section{The stability analysis and control transmission of mathematical model for Ebola Virus}

\author{
Muhammad Tahir ${ }^{1, *}$, Gul Zaman ${ }^{2}$, Syed Inayat Ali Shah ${ }^{1}$, Sher Muhammad ${ }^{1}$, Syed Asif Hussain ${ }^{1}$ and \\ Mohammad Ishaq ${ }^{1}$ \\ 1 Department of Mathematics, Islamia College Peshawar, 25000, K.P.K Pakistan. \\ 2 Department of Mathematics, University of Malakand, Chakdara District Lower Dir, K.P.K Pakistan. \\ * Correspondence: tahirshah08@yahoo.com
}

Received: 23 July 2019; Accepted: 25 November 2019; Published: 15 December 2019.

\begin{abstract}
Mathematical modeling of infectious diseases has progressed dramatically over the past four decades and continues to flourish at the nexus of mathematics, epidemiology, and infectious diseases research. Now recognized as a valuable tool, mathematical models are being integrated into the public health decision-making process more than ever before. In this article, a mathematical model of Ebola virus which is named as SEIVR (susceptible, exposed, infected, vaccinated, recovered) model is considered. First, we formulate the model and present the basic properties of the proposed model. Then, basic reproductive number is obtained by using the next-generation matrix approach. Furthermore, the sensitivity analysis of $R_{0}$ is also discussed, all the endemic equilibrium points related to the disease are derived, a condition to investigate all possible equilibria of the model in terms of the basic reproduction number is obtained. In last, numerical simulation is presented with and without vaccination or control for the proposed model.
\end{abstract}

Keywords: Ebola virus, sensitivity analysis, reproduction number, formulation of model, endemic equilibrium points, local stability, global stability, numerical simulation through Matlab programming.

MSC: 30C45, 30C50, 30C55.

\section{Introduction}

2 4 July 2018 marks the end of the ninth outbreak of Ebola in the Democratic Republic of the Congo(DRC). The World Health Organization(WHO) congratulates the country and all those involved in ending the outbreak while urging them to extend this success to combatting other diseases in DRC. The recent Ebola virus (EBOV) epidemic in West Africa emerged around the end of 2013 in the prefecture of Guckdou in Guinea [1] and caused at least 11,310 deaths among 28,616 recorded cases in Guinea, Sierra Leone, and Liberia [2]. It has been argued that the West African EBOV epidemic illustrated problems in the early detection of, and rapid response to, infectious disease outbreaks of public health importance. For the past decade, researches have been conducted in laboratories to better understand the biology and potential therapies of Ebola virus (EBOV) [3]. However, field-based research in high risk populations such as impoverished villages much progress has not been accomplished. For instance, there have been outbreaks in the Democratic Republic of Congo in 2007, 2008 and in Uganda in 2007 [4].

Mathematical models have been used extensively to study the dynamics of EBOV transmission [5]. Tahir et al. [6] presented mathematical model for the Ebola virus. A similar mathematical model was presented recently in [7]. Another recent mathematical model on the Ebola virus was studied in [8]. Ebola virus is one of the four ebolaviruses known to cause disease in humans. It has the highest case-fatality rate of these ebolaviruses, averaging $83 \%$ since the first outbreaks in 1976, although fatality rates up to $90 \%$ have been recorded in one outbreak (200203). There have also been more outbreaks of the Ebola virus than of any other ebolavirus. In 1976 the first Ebola virus was found of the Marburg virus [9,10]. In the mean while another team found Ebola virus, from Ebola River where this river was first considered to be near to the Republic of the Congo [9-11]. A mathematical model Prevention strategy for superinfection mathematical model tuberculosis and HIV associated with AIDS was recently presented [12]. 
The name Ebola virus is derived from the Ebola River, a river that was at first thought to be in close proximity to the area in the Democratic Republic of Congo. The incubation period, that is, the time interval from infection with the virus symptoms is 2 to 21 days. Humans are not infectious until they develop symptoms. The family of the related virus included (1) Cuevavirus,(2) Marburgvirus, and (3) Ebolavirus. Majority of human death occurred by Ebola virus and in West Africa and it becomes epidemic in 2013 to 2015 [13]. Some cases reported out from West Africa, all infected are foreign travelers who exposed to affected regions while later they showed Ebola fever symptoms when reached to destinations [14]. In this period the virus caused near about 286,16 are suspected while 113,10 exact and confirmed deaths cases [15]. The Ebola virus spread in many countries, which start in Guinea and move across Liberia and Sierra Leone.

The Ebola virus also spreads by the human to human contacts like secretions, blood, body fluids of the infected individuals, surfaces and the materials of infected (that is) cloth and bedding. The virus causes serious acute illness and becomes fatal if the patient takes no treatment. The Ebola virus causes an acute, serious illness which is often fatal if untreated. Pathogen genome sequencing is also being used to assist with the identification of unknown infection sources and transmission chains, as pathogen genomes contain valuable information that complements contact tracing efforts. In the case of Ebola, Arias et al. [4] demonstrated that rapid outbreak sequencing in locally established sequencing facilities can identify transmission chains linked to sporadic cases. In addition to identifying specific transmission pathways, pathogen genome analysis can also shed light on the origins, evolution and transmission dynamics of a pathogen during an epidemic [16]. Early in the EBOV epidemic analysis such as those by Gire et al.[17] demonstrated that the virus entered the human population in the late 2013 and crossed from Guinea to Sierra Leone in May 2014 through the sustained human-to-human transmission.

In this article, we directed as follows: In Section 2, the model formulation has been illustrated. In Section 3, the reproductive number is derived, and its sensitivity analysis is given in the Section 4 . Next endemic equilibrium points are derived and the local stability analysis is shown stable at disease free, as well as, at endemic equilibrium in Section 5. Further we derived the global stability of the model with the help of Lyapunov function at disease free, and at endemic equilibrium in Section 6. Finally, we have shown numerically result by $R K_{4}$ method and Matlab programming in Section 7 and conclude our paper in Section 8 .

\section{Model formulation and method}

Ebola SEIVR (susceptible, exposed, infected, vaccinated, recovered) mathematical model [18,19] is defined as:

$$
\left.\begin{array}{c}
S^{\bullet}=\frac{1}{2} \psi-\xi S-\frac{1}{\psi} \lambda S E-\epsilon_{1} S I-\epsilon_{2} S I, \\
E^{\bullet}=\lambda S E-\omega E I-\left(\mu_{1}+\mu_{2}\right) E \\
I^{\bullet}=\omega E I+\epsilon_{1} S I+\epsilon_{2} S I-\frac{1}{\zeta} \eta I R-\left(\phi_{1}+\phi_{2}\right) I, \\
V^{\bullet}=\left(\phi_{1}+\phi_{2}\right) I-\zeta V \\
R^{\bullet}=\frac{1}{\zeta} \eta I-\delta R .
\end{array}\right\}
$$

along with the following initial conditions:

$$
[S(0), E(0), I(0), V(0), R(0)] \geq 0 .
$$

Here, $S$ represent susceptible individuals, $E$ shows exposed individuals, $I$ represent infected $V$ represent the vaccinated individuals, $R$ represent recovered individuals, $\psi$ and $\xi$ represent new birth rate and death rate in susceptible individuals, $\epsilon_{1}$ represent infection transmission rate from susceptible to infected individuals through wild animals infection, and $\epsilon_{2}$ represent the infection transmission rate from susceptible individual to infected individuals through domestic animals, $\lambda$ represent infection transmission rate from susceptible to exposed individuals, $\omega$ represent infection transmission rate from exposed individual to infected individuals, $\eta$ represented the rate of recovery in recovered individuals, $\mu_{1}$ and $\mu_{2}$ represent natural death rate and infectious death rate in exposed individuals, $\phi_{1}$ and $\phi_{2}$ representing natural and infectious death rate of infected individuals respectively. We represent the total population of the model (1) as below,

$$
B(t)=S+E+I+V+R,
$$


which will be written as,

$$
\frac{d B(t)}{d t}=\frac{d S}{d t}+\frac{d E}{d t}+\frac{d I}{d t}+\frac{d V}{d t}+\frac{d R}{d t} .
$$

Using values from model (1), we get the following result

$$
\frac{d B(t)}{d t}=\psi-\xi S-\left(\mu_{1}+\mu_{2}\right) E-\eta I R-\left(\phi_{1}+\phi_{2}\right) I+\eta I-\zeta V-\delta R .
$$

From Equation (2), we have

$$
\frac{d B(t)}{d t} \leq \psi-\xi S
$$

Clearly

$$
\lim _{t \rightarrow \infty} \sup B \leq \frac{\psi}{\xi} .
$$

For the study of biological purpose, the feasible and sufficient region for model (1) is denoted by $\Re$ and defined as:

$$
\Re=\left\{(S, E, I, V, R) \in R_{+}^{5}, B \leq \frac{\psi}{\zeta}\right\} .
$$
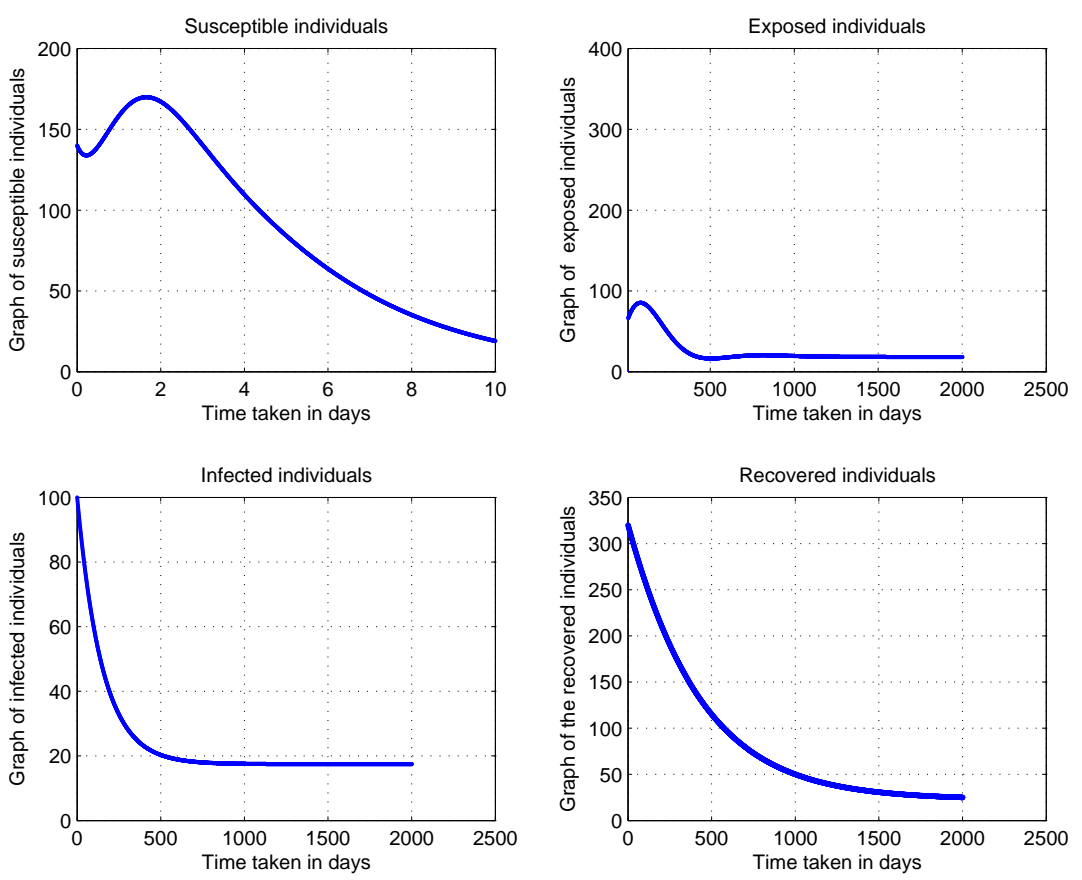

Figure 1. The plot shows the Ebola virus behavior.

\section{3. $R_{0}$-the reproduction number of model}

In many epidemiological models, the basic reproduction number is one of the key values that can predict whether the infectious disease will spread into a population or die out. The basic reproduction number is the average rate of secondary infectious cases when one infectious individual is introduced in a susceptible population. In this section we used the concept of next generation matrix method which was developed by Driessche et al. [20]. For this we divide the system into infectious class "F" and non infectious class "V" as under,

$$
F=\left[\begin{array}{c}
\lambda S E-\omega E I \\
\omega E I+\epsilon_{1} S I+\epsilon_{2} S I-\eta I R
\end{array}\right]
$$


and

$$
V=\left[\begin{array}{c}
\left(\mu_{1}+\mu_{2}\right) E \\
\left(\phi_{1}+\phi_{2}\right) I
\end{array}\right]
$$

Now to find Jacobian $\bar{F}$ and $\bar{V}$, we processed as:

$$
\begin{gathered}
\bar{F}=\left[\begin{array}{cc}
\lambda S-\omega I & -\omega E \\
\omega I & \omega E+\epsilon_{1} S+\epsilon_{2} S-\eta R
\end{array}\right] . \\
\bar{V}=\left[\begin{array}{cc}
\mu_{1}+\mu_{2} & 0 \\
0 & \phi_{1}+\phi_{2}
\end{array}\right] .
\end{gathered}
$$

Therefore, the reproductive number $R_{0}$ of our model (1) is given as:

$$
R_{0}=\frac{\left(\epsilon_{1}+\epsilon_{2}\right) \psi}{\xi\left(\phi_{1}+\phi_{2}\right)}
$$

Table 1. Sensitivity Analysis of Chosen Parameters $R_{0}$

\begin{tabular}{||ccc||}
\hline Parameter & Sensitivity Index & Value \\
\hline \hline New rate & $S_{\psi}$ & +1.0001 \\
Ebola treatment rate & $S_{\xi}$ & +0.8087 \\
Rate through class change & $S_{\epsilon_{2}}$ & -0.7687 \\
Exposed individuals treatment & $S_{\lambda}$ & -0.7761 \\
Modified parameter & $S_{\omega}$ & +0.1245 \\
Rate throuhg individual left class & $S_{\xi}$ & -0.9011 \\
Modified parameter & $S_{\psi}$ & +0.8315 \\
\hline
\end{tabular}

The Table 1 shows that their are two influences parameters involve on the rate of reproductive number, i.e, positive and negative. In addition, $\psi, \xi, \epsilon_{2}$ and $\omega$ have positive influences while $\epsilon_{1}, \lambda$ and $\eta$ have negative effect on the rate of reproductive number. From this we describe that, increasing or decreasing $10 \%$ will increase or decrease the rate of reproductive number $10 \%, 8.087 \%, 1.245 \%$ and $8.315 \%$ are given in Figures showing different images of reproductive number $R_{0}$ and 6 . On the other side, we see that the parameters index by, $\omega$, $\epsilon_{2}$ and $\xi$ describe that increase its values $10 \%$ should decrease it $10 \%$ reproductive number upto $7.687,7.761$ and 9.011 given in Figures 2, 3 and 4.

Now, to control Ebola infection, we need to focus on parameter $\psi$ which have highest sensitivity index 1.0000 , which means decreasing its value $10 \%$ will decrease the rate of reproductive number by $10 \%$ defined in [1].

\section{Endemic equilibrium points with related sketch of the model}

Now, we find the endemic equilibrium points which also play important role in any epidemiological model. The disease-free equilibrium points results to be locally asymptotically stable if the reproduction number $\left(R_{0}\right)$ is less than unity, that is 1 while the endemic equilibrium points is locally asymptotically stable if such a number exceeds unity that is greater then 1 . Following are the endemic equilibrium points of the concern model:

$$
\begin{aligned}
E^{*} & =-\frac{\xi \lambda}{} \\
I^{*} & =\frac{\lambda}{\omega} S-\frac{1}{\omega}\left(\mu_{1}+\mu_{2}\right), \\
R^{*} & =\frac{\eta}{\delta}\left(\frac{\lambda}{\omega} S-\frac{1}{\omega}\left(\mu_{1}+\mu_{2}\right)\right),
\end{aligned}
$$


The plot shows sensitivity analysis of different parameters of the reproductive number R0.
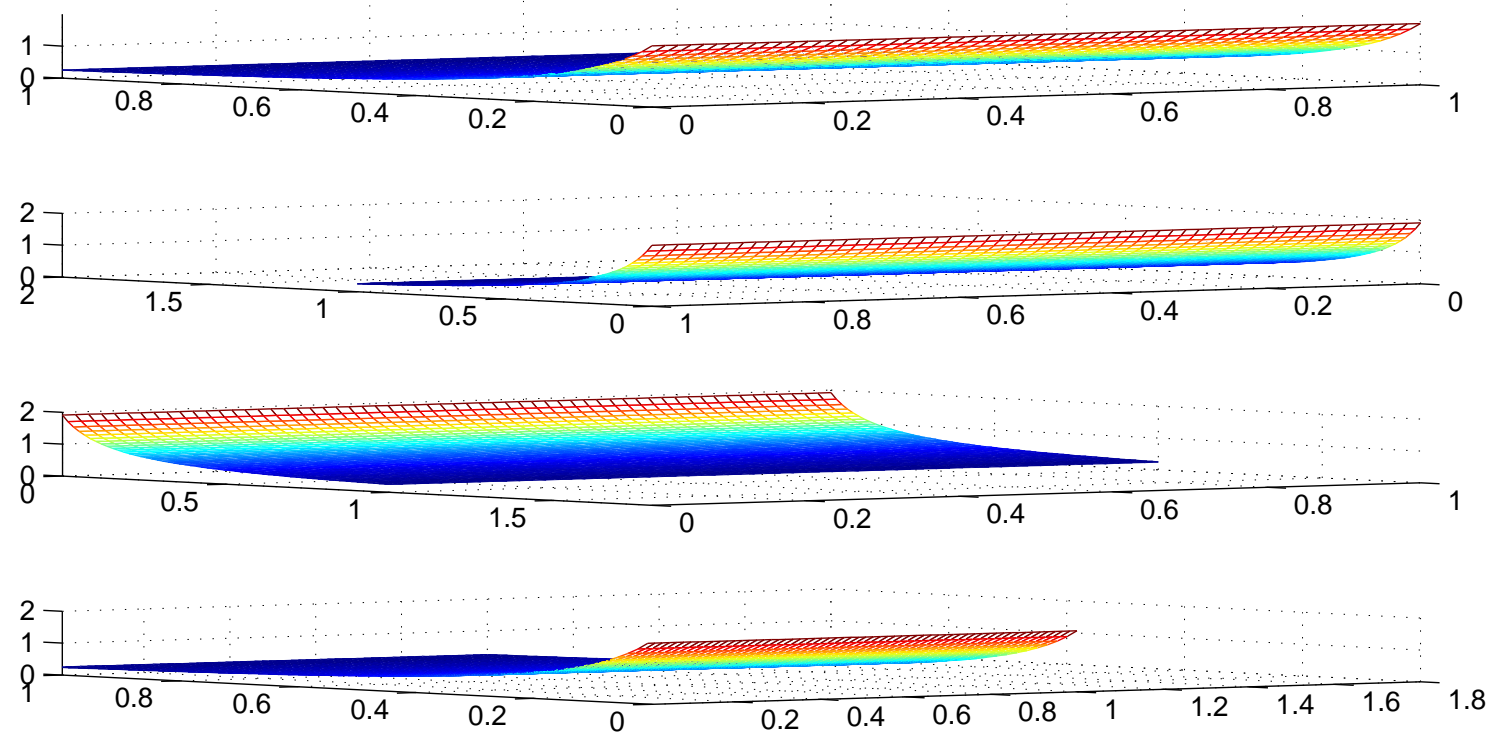

Figure 2. The plot shows sensitivity analysis of different parameters of the reproductive number $R_{0}$.

where the value of the term $S^{*}$ is given below,

$$
S^{*}=\frac{\delta \omega^{2} \xi^{2}+\lambda \delta \omega \xi\left(\phi_{1}+\phi_{2}\right)-\lambda \delta^{2}\left(\mu_{1}+\mu_{2}\right)}{\lambda\left(\delta \omega \xi\left(\epsilon_{1}+\epsilon_{2}\right)-\lambda \eta^{2}\right)} .
$$

\section{Local stability analysis of the proposed model}

\subsection{Local stability analysis at disease free equilibrium}

The local stability analysis at disease free equilibrium of the model (1), are $K F_{e}=\{S, E, I, R\}$, which implies in disease free form as $K F_{e}=\{\psi / \xi, 0,0,0\}$. Thus, we processed by the following Jacobian matrix at $K F_{e}$ :

$$
K\left(D E_{e}\right)=\left[\begin{array}{cccc}
\xi & -\lambda S & -\left(\epsilon_{1}+\epsilon_{2}\right) S^{0} & 0 \\
0 & \lambda S^{0}-\left(\mu_{1}+\mu_{2}\right) & 0 & 0 \\
0 & 0 & \left(\epsilon_{1}+\epsilon_{2}\right) S^{0}-\left(\phi_{1}+\phi_{2}\right) & 0 \\
0 & 0 & 0 & \delta\left\{\left(\epsilon_{1}+\epsilon_{2}\right) S^{0}-\left(\phi_{1}+\phi_{2}\right)\right\}
\end{array}\right]
$$

Thus for local stability analysis of disease free equilibria, we have the following Theorem 1.

Theorem 1. At disease free equilibrium $K F_{e}=\{\psi / \xi, 0,0,0\}$. If $R_{0}<1$, then the concern model (1) is locally asymptotically stable, while if $R_{0}>1$, the model (1) is unstable.

Proof. We have the following eigenvalues from Jacobian matrix $J\left(K E_{e}\right)$ :

$$
\begin{aligned}
& \lambda_{1}=-\xi \\
& \lambda_{2}=\lambda S^{0}-\left(\mu_{1}+\mu_{2}\right), \\
& \lambda_{3}=\left(\epsilon_{1}+\epsilon_{2}\right) S^{0}-\left(\phi_{1}+\phi_{2}\right), \\
& \lambda_{4}=\delta\left\{\left(\epsilon_{1}+\epsilon_{2}\right) S^{0}-\left(\phi_{1}+\phi_{2}\right)\right\} .
\end{aligned}
$$


The plot shows sensitivity analysis of different parameters of the reproductive number R0.
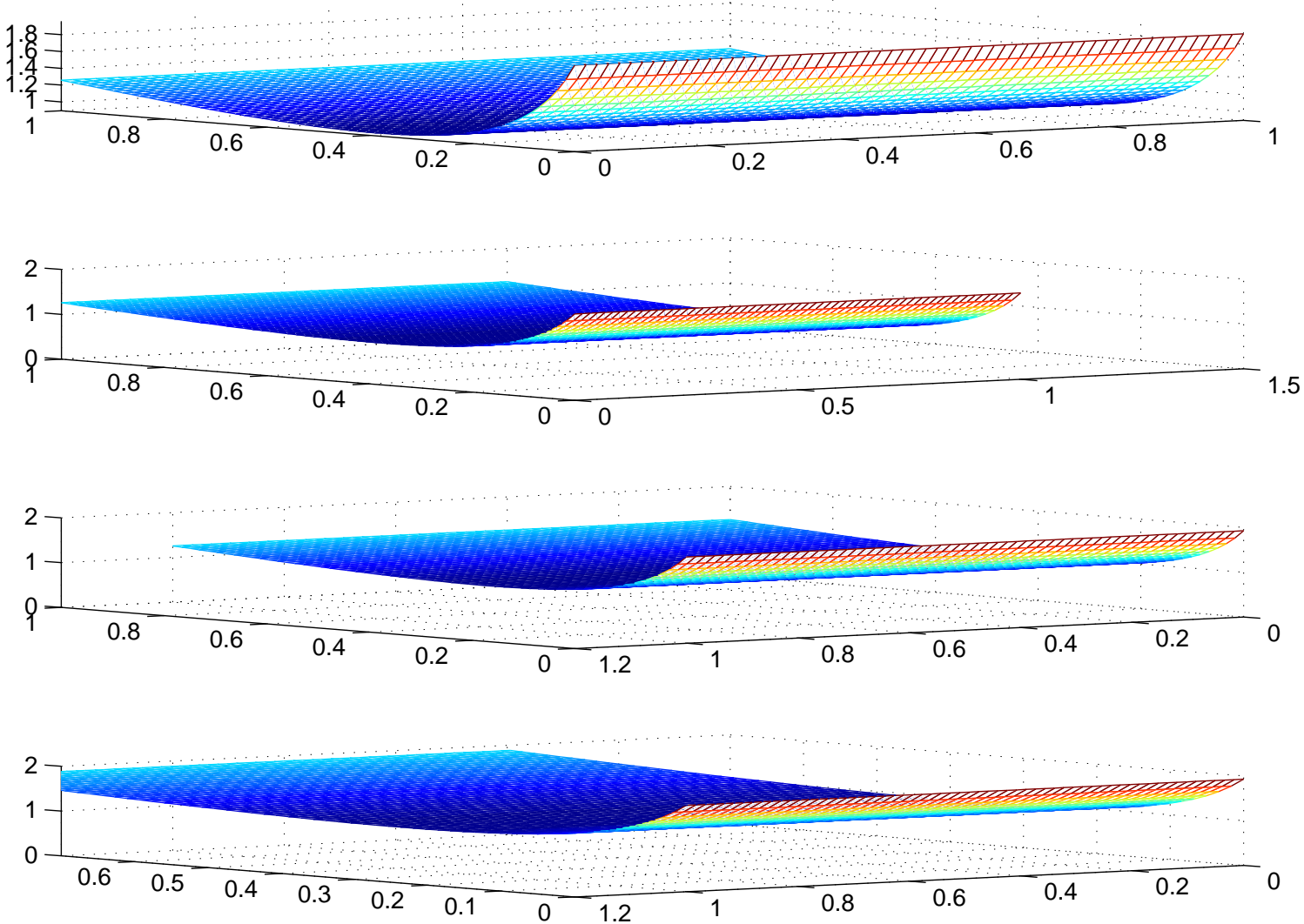

Figure 3. The plot shows sensitivity analysis of different parameters of the reproductive number $R_{0}$.
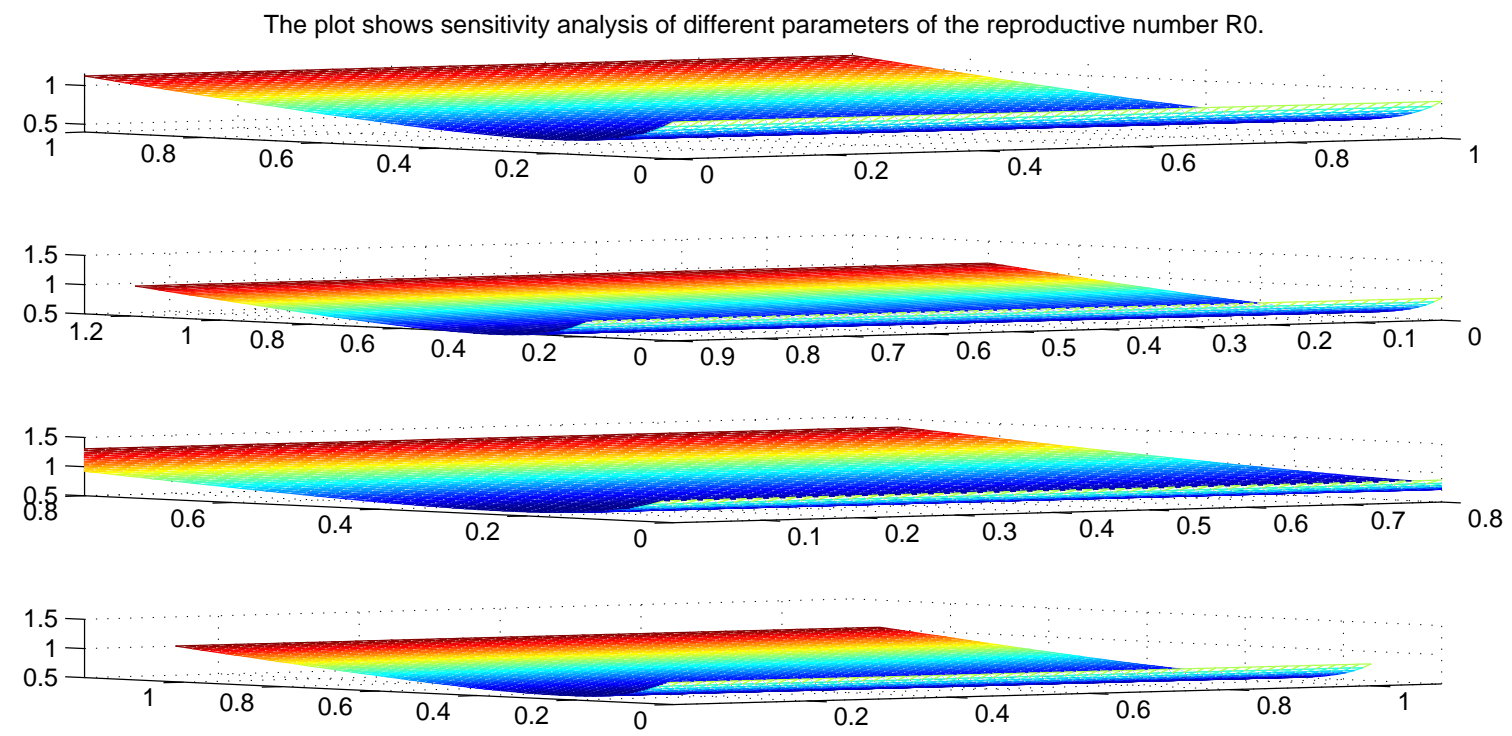

Figure 4. The plot shows sensitivity analysis of different parameters of the reproductive number $R_{0}$. 

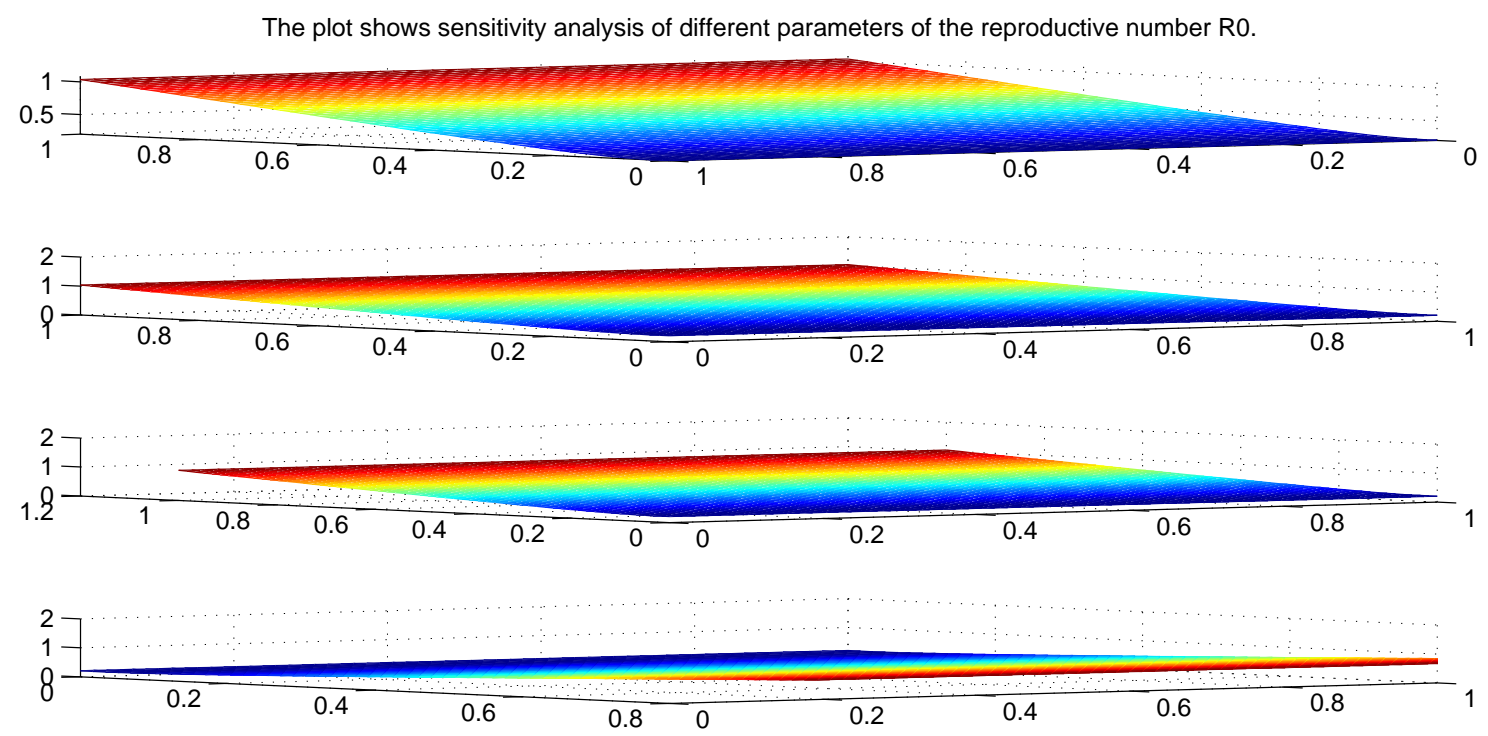

Figure 5. The plot shows sensitivity analysis of different parameters of the reproductive number $R_{0}$.

It is clear from Equation (5), $\lambda_{1}=-\xi<0$. Taking Equation (6) $\lambda_{2}=-\left\{\left(\mu_{1}+\mu_{2}\right)-\psi\right\}$. It implies that $\lambda_{2}<0$ if and only if $\left(\mu_{1}+\mu_{2}\right)>\psi$. From Equation (7) (that is) $\lambda_{3}=\left(\epsilon_{1}+\epsilon_{2}\right) S_{h}^{0}-\left(\phi_{1}+\phi_{2}\right)$. So clearly $\lambda_{3}=R_{0}-1$. So $\lambda_{3}=<0$ iff $R_{0}<1$. From Equation (8) $\lambda_{4}=-\delta\left\{1-R_{0}\right\}<0$ if and only if $R_{0}<1$, which complete the proof.

\subsection{Local Stability Analysis At Endemic Equilibrium}

For local stability analysis at endemic equilibrium, we have the following result.

Theorem 2. Local asymptotical stability at endemic equilibrium, will hold if $R_{0}>1$ for model (1) that is, at $K E_{e}=$ $\left\{S^{*}, E^{*}, I^{*}, R^{*}\right\}$ and unstable if $R_{0}>1$.

Proof. For stability analysis at endemic equilibrium, consider the $4 \times 4$ matrix as:

$$
K E_{e}=\left(\begin{array}{cccc}
-\xi+\lambda E^{*}+K_{1} & 0 & -\left(\epsilon_{1}+\epsilon_{2}\right) S^{*} & 0 \\
0 & -K_{1} K_{2} & K_{4} & 0 \\
0 & 0 & \left(\omega K_{4} K_{1}\right) I^{*}+K_{1} K_{2} K_{5} & -\left(\eta K_{1}^{2} K_{2}\right) I^{*} \\
0 & 0 & 0 & K_{6}
\end{array}\right)
$$

where,

$$
\begin{aligned}
& K_{1}=\left(\epsilon_{1}+\epsilon_{2}\right) I^{*}, \\
& K_{2}=\lambda S^{*}-\omega I^{*}-\left(\mu_{1}+\mu_{2}\right), \\
& K_{3}=\omega E^{*}+\left(\epsilon_{1}+\epsilon_{2}\right) S^{*}-\eta R^{*}-\left(\phi_{1}+\phi_{2}\right), \\
& K_{4}=-\left\{\lambda\left(\epsilon_{1}+\epsilon_{2}\right) S^{*}+\omega K_{1}\right\} E^{*}, \\
& K_{5}=-\left\{\left(K_{1} K_{2}\right)+\left(\epsilon_{1}+\epsilon_{2}\right) I^{*}\left(\epsilon_{1}+\epsilon_{2}\right) S^{*}\right\}, \\
& K_{6}=-\delta\left(\omega K_{1} K_{4} I^{*}+K_{1} K_{2} K_{5}\right)+\eta^{2}\left(K_{1}^{2} K_{2}\right) I^{*} .
\end{aligned}
$$


Thus for endemic equilibrium, we get

$$
\begin{aligned}
& \lambda_{1}^{*}=-\xi+\lambda E^{*}+K_{1} \\
& \lambda_{2}^{*}=-K_{1} K_{2} \\
& \lambda_{3}^{*}=\left(\omega K_{4} K_{1}\right) I_{e}^{*}+K_{1} K_{2} K_{5} \\
& \lambda_{4}^{*}=K_{6} .
\end{aligned}
$$

Now, from Equation (9) $\lambda_{1}^{*}=-\left\{\xi+\xi E^{*}+\left(\epsilon_{1}+\epsilon_{2}\right) I^{*}\right\}$, so $\lambda_{1}<0$ iff $\left(\phi_{1}+\phi_{2}\right)+\omega>\left(\mu_{1}+\mu_{2}\right)$ and $\left(\phi_{1}+\phi_{2}\right)+\omega>\eta^{2}$. Now by using Equation (10), $\lambda_{2}^{*}=-K_{1} K_{2}<0$ if and only if $\lambda<1$ and $\lambda>\omega$. Now by checking the value of $\lambda_{3}^{*}$, from Equation (11), we observed that $\lambda_{3}^{*}=\left(\omega K_{4} K_{1}\right) I_{e}^{*}+K_{1} K_{2} K_{5}<0$ iff $\left\{\lambda\left(\epsilon_{1}+\epsilon_{2}\right) S_{h}^{*}+K_{1} \omega\right\} \omega E^{*} I^{*}>\omega I^{*}+\left\{\left(\mu_{1}+\mu_{2}\right)\left(k_{1} k_{2}\right)\left(\epsilon_{1}+\epsilon_{2}\right)^{2}-\lambda\right\} S^{*}$. By performing some calculation, we observed that $\lambda_{3}<0$. Taking Equation (12) and performing some calculation, we have $\lambda_{4}<0$ if and only if $\omega K_{4} I^{*}+K_{2} K_{5}>\eta^{2} K_{1} K_{2} I^{*}$. Clearly local stability analysis at endemic equilibrium is asymptotically stable for system (1) which completed the proof.

\section{Global stability analysis of the proposed model}

In this section, we discuss the global stability analysis of the problem. There is a power full tool Lyapunov function, that is used for the global stability analysis, hence to check the global stability analysis of the model (1), we construct a Lyapunov function [21,22]. We have two cases: (1) global stability analysis at disease free equilibrium and (2) global stability analysis at endemic equilibrium.

\subsection{Global stability analysis at disease free equilibrium}

Theorem 3. For system (1), if $R_{0} \leq 1$, then Globally asymptotically stability will hold for disease free equilibrium if $S=S 0$ and unstable for $R_{0}>1$.

Proof. To show global stability at disease free equilibrium of the model (1), considered the following Lyapunov function:

$$
U(S, E, I, R)=\frac{1}{3}\left(S-S^{0}+E-E^{0}+I-I^{0}\right)^{3}
$$

Obviously the'above function is greater then zero at disease free equilibrium and equal to zero at $S=S^{0}$, and $E=I=R=0$. Differentiating $U(S, E, I, R)$ with respect to $t$, we obtain the following result:

$$
\frac{d U}{d t}(S, E, I, R)=\left(S-S^{0}+E-E^{0}+I-I^{0}\right)^{2} \psi-\lambda S+\lambda S E-\left(\mu_{1}+\mu_{2}\right) E-\eta I R-\left(\phi_{1}+\phi_{2}\right) I .
$$

After some simplification, we get

$$
\frac{d U}{d t}(S, E, I, R)=-\left(S-S^{0}+E-E^{0}+I-I^{0}\right)(K-Q) .
$$

Clearly Equation (13) is less then zero if and only if $K>Q$, where

$$
K=\psi E+\left(\mu_{1}+\mu_{2}\right) E+\left(\eta R+\left(\phi_{1}+\phi_{2}\right)\right) I,
$$

and

$$
Q=(1+E) \psi
$$

Here we see that $\frac{d U}{d t}(S, E, I, R)=0$ if and only if $S=S^{0}, E=E^{0}, I=I^{0}$, and $R=R^{0}$ while $\frac{d U}{d t}(S, E, I, R)<$ 0 iff $K>Q$. Then the disease free equilibrium is globally asymptotically stable. 


\subsection{Global stability analysis at endemic equilibrium}

Theorem 4. For globally asymptotically stability, if $R_{0}>1$, then the endemic equilibrium of model (1) is stable and $S=S^{*}, E=E^{*}, I=I^{*} R=R^{*}$ and unstable, if $R_{0}<1$.

Proof. For global stability analysis at endemic equilibrium, we define

$$
Q(S, E, I, R)=\frac{1}{2}\left(S-S^{*}\right)^{2}+\frac{1}{2}\left(I-I^{*}\right)^{2},
$$

we have $Q(S, E, I, R)>0$ and it equal to zero at $S=S^{*}, E=E^{*}, I=I^{*}$. Differentiating $Q(S, E, I, R)$ with respect to $t$ we get,

$$
\frac{d Q}{d t}(S, E, I, R)=\left(S-S^{*}+I-I^{*}\right)\left(\frac{d}{d t} S+\frac{d}{d t} I\right),
$$

Putting values from model (1) in above, we obtain

$$
\frac{d Q}{d t}(S, E, I, R)=-\left(S-S^{*}+I-I^{*}\right)\left(\eta R+\phi_{1}+\phi_{2}-\omega E\right) I .
$$

Hence we have $\frac{d Q}{d t}(S, E, I, R)=0$ if and only if $S=S^{*}, E=E^{*}, I=I^{*}$ and $R=R^{*}$. Also $\frac{d Q}{d t}(S, E, I, R)<0$, iff $\eta R+\phi_{1}+\phi_{2}>\omega E$, hence endemic equilibria is globally asymptotically stable for model (1). So the proof is completed.
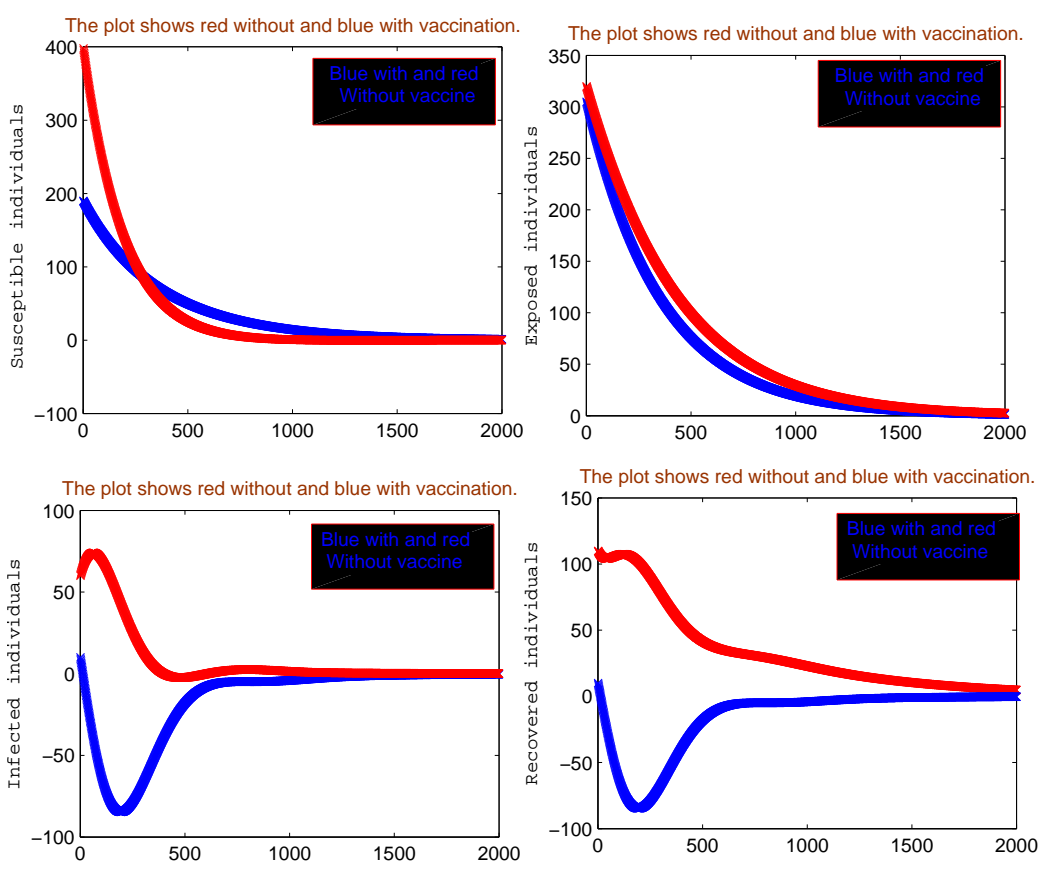

Figure 6. The plot shows red without and blue with vaccination.

\section{Numerical simulation and discussion}

In this section, we present numerical interpretation of the proposed model with the help of Matlab programming. Numerical results given in Figure 1 shows simple graph of the model having no vaccination used yet, Figures 2-5 represents different behaviour of the reproductive number $R_{0}$, Figure 6 with and without vaccination in population, while Figures 7 and 8 shows graph with and without vaccine and also the area infected graph if no vaccination taken. 
We present the following Table 2. By using the parameters value, from Table 2, non-negative initial population sizes and from different time interval, we obtain the simulation Figure 6, which represents that there are always susceptible $S(t)$ and recovered $R(t)$ population which quickly recovered with vaccination, while the remaining individuals populations i.e. exposed $E(t)$, and infected $I(t)$ individuals respectively shown the exposed individuals recovery is very slow without vaccination, while with vaccination, a rapid effeteness occur in there health condition. Similarly if we do not provide vaccine to the infected class we see from simulation their graph is going high but vaccination rapidly cover their problem. Also the population in the model is represented by area graph. In the area graph the less area shows less infection while more cover area graph shows great infection in any time in any population.

All values taken fixed in the Table 2 . In Figure 6 the simulation are presented with and without vaccination to the population, while Figure 7 and 8 shows effected area if no vaccination used.

Table 2. Values of Parameters

\begin{tabular}{||crc||}
\hline Notation & Description of Parameter & Value \\
\hline \hline$S$ & Susceptible individuals population & $00-2000$ \\
$E$ & Exposed individuals population & $00-2000$ \\
$I$ & Infected individuals population & $00-2000$ \\
$R$ & Recovered individuals population & $00-2000$ \\
$\psi$ & New birth rate in susceptible individuals & 0.6321 \\
$\lambda$ & Transmission rate from susceptible to exposed individuals & 0.2877 \\
$\omega$ & Transmission rate from exposed to infected individuals & 0.7613 \\
$\eta$ & Transmission rate from infected to recover individuals & 0.4389 \\
$\epsilon_{1}$ & Individuals get wild animals infection from susceptible to infected & 0.1234 \\
$\epsilon_{2}$ & Individuals get domestic animals infection from susceptible to infected & 0.2431 \\
$\mu_{1}$ & Natural death rate of exposed individuals & 0.9704 \\
$\mu_{2}$ & Infectious death rate of exposed individuals & 0.0432 \\
$\phi_{1}$ & Natural death rate of infected individuals & 0.2006 \\
$\phi_{2}$ & Infectious death rate of infected individuals & 0.0656 \\
$\delta$ & Natural death rate of recover individuals & 0.6704 \\
\hline
\end{tabular}
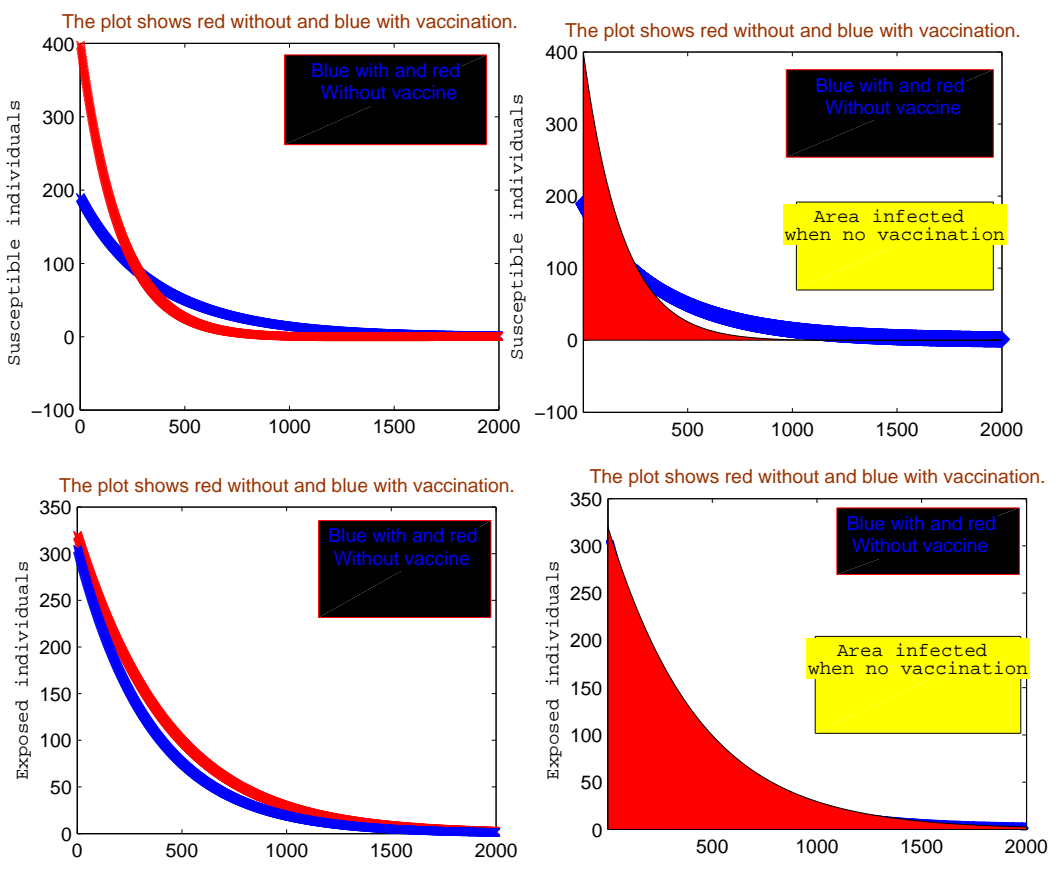

Figure 7. The plot shows red without and blue with vaccination. 

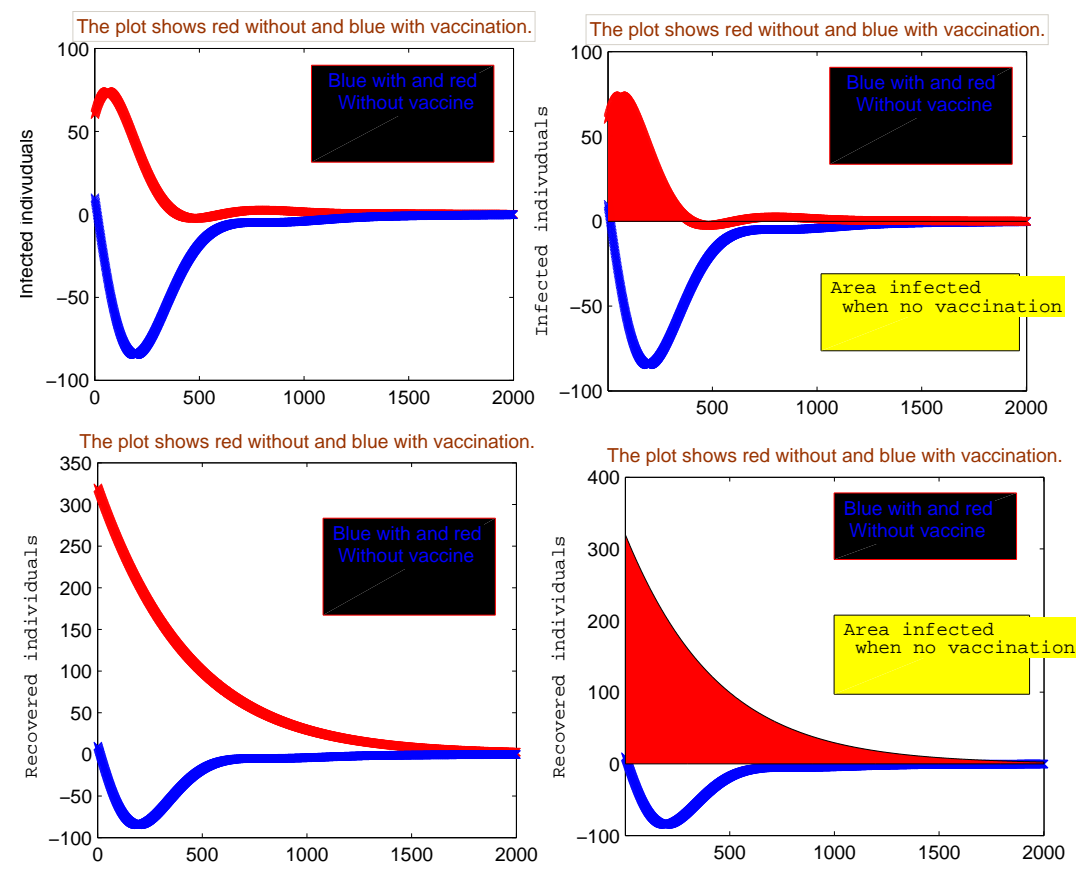

Figure 8. The plot shows red without and blue with vaccination.

\section{Conclusion}

A mathematical epidemic model SEIR of the Ebola virus is considered where the transmissibility agent is considered animal at any time " $\mathrm{t}$ " in the population. First we formulated the model according to there infectious classes, and by next-generation matrix approach, we find reproductive number (that is) $R_{0}$ with biological feasible region. Also we discussed the reproductive number sensitivity indices by showing different behavior of $R_{0}$. After we discussed endemic equilibrium points of the model. Then according to the reproductive number we discussed the local stability and global stability at disease free equilibrium and at endemic equilibrium and shown stable. The global stability at both respects is discussed with the help of Lyapunov function. Finally, we obtained numerical solution of compartmental mathematical model by the using Matlab program. Also we obtained the area involved we no vaccination taken. The figures show first column of the with and without vaccination population while the second column shows the infected population area of the model. The graph approaches represent new idea for the scientists in future. In the area graph, the less area shows less infection while more cover area graph shows great infection at any time in any population.

Author Contributions: All authors contributed equally to the writing of this paper. All authors read and approved the final manuscript.

Conflicts of Interest: "The authors declare no conflict of interest."

\section{References}

[1] Lamb, R. A. (2013). Fields Virology. In Fields Virology (eds Knipe, D. M. and Howley, P. M. ) 880-884 (Lippincott Williams and Wilkins, 2013).

[2] Feldmann, H., Sanchez, A., \& Geisbert, T. (2013). Fields Virology. In Fields Virology (eds Knipe, D. M. and Howley, P. M. ) 923-956 (Lippincott Williams and Wilkins, 2013).

[3] Blackwood, J. C., \& Childs, L. M. (2016). The role of interconnectivity in control of an Ebola epidemic. Scientific reports, 6, 29262.

[4] Arias, A., Watson, S. J., Asogun, D., Tobin, E. A., Lu, J., Phan, M. V., ... \& Caddy, S. (2016). Rapid outbreak sequencing of Ebola virus in Sierra Leone identifies transmission chains linked to sporadic cases. Virus evolution, 2(1).

[5] Lau, M. S., Dalziel, B. D., Funk, S., McClelland, A., Tiffany, A., Riley, S., ... \& Grenfell, B. T. (2017). Spatial and temporal dynamics of superspreading events in the 2014-2015 West Africa Ebola epidemic. Proceedings of the National Academy of Sciences, 114(9), 2337-2342. 
[6] Tahir, M., Inayat Ali Shah, S., Zaman, G., \& Muhammad, S. (2018). Ebola virus epidemic disease its modeling and stability analysis required abstain strategies. Cogent Biology, 4(1), 1488511.

[7] Tahir, M., Shah, S. I. A., Zaman, G., \& Khan, T. (2019). A Dynamic Compartmental Mathematical Model Describing The Transmissibility Of MERS-CoV Virus In Public. Journal of Mathematics, 51(4), 57-71.

[8] Tahir, M., Anwar, N., Shah, S. I. A., \& Khan, T. (2019). Modeling And Stability Analysis of Epidemic Expansion Disease Ebola Virus with Implications Prevention in Population. Cogent Biology, (just-accepted), 1619219.

[9] Pattyn, S., Jacob, W., Van der Groen, G., Piot, P., \& Courteille, G. (1977). Isolation of Marburg-like virus from a case of haemorrhagic fever in Zaire. Lancet, 573-574.

[10] Bowen, E. T. W., Platt, G. S., Lloyd, G., Baskerville, A., Harris, W. J., \& Vella, E. E. (1977). Viral haemorrhagic fever in southern Sudan and northern Zaire. Preliminary studies on the aetiological agent. Lancet, 571-573.

[11] Kuhn, J. H., Becker, S., Ebihara, H., Geisbert, T. W., Johnson, K. M., Kawaoka, Y., ... \& Palacios, G. (2010). Proposal for a revised taxonomy of the family Filoviridae: classification, names of taxa and viruses, and virus abbreviations. Archives of virology, 155(12), 2083-2103.

[12] Tahir, M., Shah, S. I. A., \& Zaman, G. (2019). Prevention strategy for superinfection mathematical model tuberculosis and HIV associated with AIDS. Cogent Mathematics E Statistics, 6(1), 1637166.

[13] World Health Organization, \& World Health Organization. (2015). Ebola data and statistics. WHO. Published March.

[14] Khan, M. A., Islam, S., \& Arif, M. (2013). Transmission model of hepatitis B virus with the migration effect. BioMed research international, 2013.

[15] Reardan, S. (2014). The first nine months of the epidemic and projection, Ebola virus disease in west Africa. archive of Ebola Response Team. New England Journal of Medicine, 511(75.11), 520.

[16] Holmes, E. C., Dudas, G., Rambaut, A., \& Andersen, K. G. (2016). The evolution of Ebola virus: Insights from the 2013Ü2016 epidemic. Nature, 538(7624), 193.

[17] Gire, S. K., Goba, A., Andersen, K. G., Sealfon, R. S., Park, D. J., Kanneh, L., ... \& Wohl, S. (2014). Genomic surveillance elucidates Ebola virus origin and transmission during the 2014 outbreak. science, 345(6202), 1369-1372.

[18] Anderson, R. M., Anderson, B., \& May, R. M. (1992). Infectious diseases of humans: dynamics and control. Oxford university press.

[19] Keeling, M. J., \& Rohani, P. (2011). Modeling infectious diseases in humans and animals. Princeton University Press.

[20] Van den Driessche, P., \& Watmough, J. (2002). Reproduction numbers and sub-threshold endemic equilibria for compartmental models of disease transmission. Mathematical biosciences, 180(1-2), 29-48.

[21] Reardan, S. (2014). The first nine months of the epidemic and projection, Ebola virus disease in west Africa. archive of Ebola Response Team. New England Journal of Medicine, 511(75.11), 520.

[22] Van den Driessche, P., \& Watmough, J. (2002). Reproduction numbers and sub-threshold endemic equilibria for compartmental models of disease transmission. Mathematical biosciences, 180(1-2), 29-48.

(c) 2019 by the authors; licensee PSRP, Lahore, Pakistan. This article is an open access article distributed under the terms and conditions of the Creative Commons Attribution (CC-BY) license (http://creativecommons.org/licenses/by/4.0/). 